\title{
Russian legal experience in using information technologies in diagnostics of reflux esophagitis
}

\author{
Anna Voevodina ${ }^{1, *}$ \\ ${ }^{1}$ Sechenov First Moscow State Medical University of the Ministry of Healthcare of the Russian Federation (Sechenov \\ University), Moscow, Russia, anny1608@mail.ru
}

\begin{abstract}
This publication focuses on a theoretical investigation of the issues of Russian experience in the application of digital technologies in the provision of medical care in the diagnosis of oesophageal hernia complicated by reflux esophagitis. The relevance of the research lies not only in the increasing number of patients diagnosed with reflux esophagitis, but also in the current digital paradigm of the entire Russian healthcare system. Despite domestic legislative attempts to legally regulate the use of digital technologies in health care through the regulatory introduction of telemedicine technology, as is most common, certain issues in the use of this digital "platform" in health care remain unresolved. The methodological basis of this study is the methods of comparative analysis, system-structural analysis and synthesis. The author has assessed the possibility of using telemedicine technologies in the context of the doctor-patient relationship, implemented in a remote format, when diagnosing a patient, including from the position of significant territorial remoteness of the patient. There is a focus on the need for a more specific legal regulation in terms of legally establishing the definition for guaranteeing the security of personal data received by participants in the consultation, processed by them when applying telemedicine technologies.
\end{abstract}

\section{Introduction}

The use of digital technology in various spheres of public life in the Russian Federation is becoming more widespread and more relevant every year. The Russian healthcare system is no exception. It consists of state, municipal and private healthcare systems. The prerequisites for the "digital" transformation of health care are not only the use of highly informative techniques, electronic medical devices and treatments, including for surgical interventions, but also the possibility of using telemedical technologies for consultation (case conference) even at the stage of primary health care. The possibility of applying telemedicine technologies in various fields of clinical medicine is one of the significant directions of digital development in health care. The doctrine notes the nonspecificity for Russian law enforcement to resolve issues relating to the settlement of electronic processes [1]. On the domestic legislative level, the issue of the possibility of using telemedicine technologies was regulated since 2017 in the Federal Law No. 323-FZ of 21 November 2011 "On the Fundamentals of Citizens' Health Protection in the Russian Federation" (hereinafter referred to as the Law on Fundamentals of Health Protection), which was amended by introducing a new legal norm (Article 36.2) devoted to the specifics of medical care provided using telemedicine technologies. Certainly, the legal regulation of the issue of telemedicine technologies is not limited to the mentioned legal act because there are other legal acts, including those of sectoral agencies, adopted to implement and enforce it. In this case, we are referring to Order No. 965n of the Ministry of Health of the Russian Federation of 30 November 2017 "On Approval of the Procedure for Organizing and Providing Medical Care Using Telemedicine Technologies" (hereinafter, Order No. $965 n)$. It is valid to suggest that there are consequences of the spread of digital technology, such as the creation of new modes and means of regulation [2].

\section{Problem Statement}

There is a variation in the content of the relationship regulated by and arising from the use of telemedicine technologies, as it involves two areas: the interaction between health care professionals, primarily related to the rapid transmission of information and exchange of medical documentation regarding the patient, and the interaction through the doctor-patient system. The latter involves resolving a rather significant range of issues related to patient diagnosis, determining the applicable treatment methods, issuing referrals to highly specialized medical institutions, monitoring and analysing data obtained from laboratory, instrumental and functional tests. However, regardless of the chosen direction of the relationship based on the use of telemedicine technologies, the actual basis for this relationship is the

\footnotetext{
*Corresponding author: anny1608@mail.ru
} 
remote interaction realized "at a distance". In the context of digital doctor-patient relationships, the main form of their implementation is the counselling services (consultation, case conference) provided online, i.e. in real time, through the use of videoconferencing, and involving direct interaction between the patient (or his legal representative) and the attending physician, medical workers (participants of the case conference), who determine the presence (absence) of any pathological abnormality in the patient based on the medical data provided electronically. Direct interaction between the participants in the consultation and the patient (or their legal representative) is possible not only through the exchange of voice information, but also through visual information. The doctrine mentions the possibility of collecting and transmitting patient information using modern telemedicine complexes [3]. Moreover, there is a position on the current expansion of telemedicine to all health services and on physician telemedicine consultations as the most well-known and widespread type of telemedicine services, while further noting the correlation of current advances in telemedicine with the advent of digital information transmission channels [4]. Despite the legal regulation of the use of telemedicine technologies in the provision of medical care based on using a "digital platform" of highspeed transmission of various information, the actualization of this method of providing medical care is certainly becoming increasingly important in view of the social processes that are emerging and taking place in society, often contributing to the remote ("distant") interaction between patient and treating physician (e.g. coronavirus infection COVID 2019). The scientific literature points to the unresolved legal issues related to the implementation of telemedicine services, which include issues of licensing and accreditation of doctors practising in different subjects of the state, requirements for diagnostics of patients, their treatment, if personal contact of doctor and patient is required, issues of legal liability [5].

\section{Research Questions}

The use of telemedicine technology in the diagnosis of oesophageal hernia complicated by reflux esophagitis is significant because of the high prevalence of these diseases in the structure of digestive diseases [6]. The doctrine has also suggested an increase in the number of patients with reflux esophagitis due to an axial hernia of the oesophageal orifice [7]. In addition, there is also a growing subject interest in investigating the dysfunctional relationship between the gastrointestinal tract and the cardiovascular system [8]. The consequences of failing to diagnose and detect this disease in time can be very negative, involving complications that put the patient's life at risk. However, timely diagnosis and detection of oesophageal hernia and associated symptoms is not possible without the use of information and technical diagnostic tools. One of the most informative and basic methods in diagnosing diseases of the oesophagus and stomach is the radiological method, which allows the most reliable examination of the topography, the anatomical structure, the functional state of the oesophagus and stomach, and gastro-esophageal reflux. There is evidence that indirect signs of oesophageal diaphragmatic hernia can be detected by noncontact examination, but with radiological data [9]. Radiological examination not only allows a diagnosis of a herniated oesophagus but also reveals cardiac insufficiency and the presence of reflux esophagitis symptoms of various kinds, while helping to visualize the oesophagus and draw conclusions about the state of the underlying gastrointestinal tract. Of course, this examination is not the only means of diagnosing the patient. The choice and tactics of diagnosis are casespecific, which in fact also predetermines the prospects of specific treatment technologies in each situation [10] However, using radiology data, a "non-contact" consultation with the patient is possible through the use of telemedicine technology.

\subsection{Evaluation of radiological data using telemedicine technology: practical relevance}

The practical implementation of a remote "digital" doctor-patient interaction when advising a patient about pathological changes and abnormalities suspected to exist in the gastrointestinal tract involves the patient first undergoing a "face-to-face" (in-person) radiological examination. Subsequently, it is interlinked with the electronic transmission of the results to the participants of the consultation for the evaluation of the medical findings reflected in the relevant documentation. The geographical features of the Russian state make it impossible to provide qualified medical care everywhere [11]. The doctrine mentions the existence of pilot telemedicine projects in the Russian Federation for patients living in remote areas and experiencing difficulties in organizing regular visits to specialists [12]. There is also a positive dynamics in the interaction between the federal research centre of the healthcare system and the clinical hospital of the subject (Cheboksary city), expressed in the provision of telemedical consultations for 38 patients and (or) medical documents of these patients in three calendar years [13].

\subsection{Safeguarding patient confidentiality in telemedicine: is it guaranteed?}

The legal basis for the implementation of telemedicine technologies, as noted, is set out in the provisions of Article 36.2 of the Law on the Fundamentals of Health Care, Order No. 965n, establishing general legal requirements on the need to ensure the confidentiality of patient data included in the patient's personal data and medical secrecy. However, it is an open question as to whether the high-speed channels for transmitting patient information are sufficiently secure, even though participants have limited remote access to video conferencing for a specific consultation and the patient is aware of the name and contacts of the operator of the 
information system used in the consultation. It is also an unresolved issue as regards the preservation of patient data in the relevant information system from the point of view of preventing "unregulated" adjustments to any information about the patient and his or her medical parameters.

\section{Purpose of the Study}

The purpose of the study is to examine the prospects of using telemedicine technologies in the diagnosis of patients with hiatal hernia of the diaphragm, complicated reflux esophagitis, and determine the further tactics of their treatment, including the position of a significant territorial remoteness of the patient, located in a sparsely populated point (administrative-territorial unit) of the Russian Federation.

\section{Research Methods}

The methodological basis of the study is the methods of comparative analysis, system-structural analysis and synthesis.

\section{Findings}

\subsection{Evaluation of radiological data using telemedicine technology: practical relevance}

There is a particular interest in optimising the diagnosis of oesophageal hernia complicated by reflux esophagitis due to the increasing prevalence of this nosological condition. The incidence of this disease is typical both for patients residing in large cities (constituent entities) of the Russian Federation and for patients residing and staying in remote (sparsely populated) areas of the Russian Federation. In the latter case, however, the level of quality and accessibility of the medical care provided (this case also requires "highly specialized" care) often does not meet the evolving needs of society and therefore its provision is sometimes extremely problematic. However, it cannot be made dependent on the patient's place of residence (location) to provide quality and timely medical care by the specialists whose expertise they need to heal. The investigated digital paradigm of remote health care delivery, implemented from the primary health care stage, represents an entirely new format of health care, enabling the use of the necessary software and hardware "kit" for patientphysician interaction. The use of telemedical technologies in the form of consultations (case conferences) based on medical data about a patient with specific symptomatology will make it possible to reasonably assess the need for, for example, surgical intervention and also determine the tactics of the patient's treatment. In this case, such actions and activities aimed at assessing the patient's condition (and possibly issuing a referral for surgery), using this digital format of interaction with a patient located in a locality remote from major cities in the Russian Federation, where the level of healthcare development is certainly higher, will be implemented by competent specialists. Thus, the practical application of telemedicine services will actually reduce the number of patient visits to outpatient clinics, minimizing the need for personal visits to the attending doctor, provided that all data on the patient's medical parameters are available electronically, if they are sufficient to make a diagnosis without the patient being present in person. It is fair to note the positive dynamics of using telemedicine technologies in the form of reducing the time a patient spends in hospital for preoperative examinations if they are needed [14], improving the quality of life of patients and reducing emergency admissions and hospitalizations [15] and allowing for consultations with competent specialists [16]). The statement about relieving the patient of the need to travel to a treatment facility that is significantly distant from their place of residence is similarly valid [17]. The administrative-territorial division of the Russian Federation and the significant geographical scale of the entire country, which implies the presence of quite remote (from large cities, including federal cities) settlements (often sparsely populated) in which the provision of specialized (and highly specialized) medical care is not always possible, justifies the incorporation of telemedical technologies in the practical medical activity.

\subsection{Safeguarding patient confidentiality in telemedicine: is it guaranteed?}

The information sent electronically about a patient, including medical records of any pathological changes, falls into the category of confidential, personal information about the patient, the free dissemination of which is prohibited by Russian law at the domestic level. The use of a digital doctor-patient system should strictly adhere to the principle of information protection of personal data on the patient. The implementation of such a digital approach is not possible without the use of the Internet telecommunication network, the availability of which gives both parties to the relationship access to a videoconference for the consultation. It seems insufficient to have a separate legal provision, referring to the prohibition of dissemination of this information without further specification of the organizational procedure for ensuring the information security of highspeed transmission channels used in telemedicine technologies. Moreover, the absence of a legal provision for the preservation of patient data in uncorrected form, uploaded to an appropriate information system to store such data, does not in fact provide the consumer of a health service with a sufficient level of guarantee of its availability in an unchanged format. It is possible that access to patients' personal data, including information that constitutes medical secrecy, may be open to a wide range of people (e.g. in the event of a technical failure resulting in an unauthorized data breach). To minimize such situations, it would be advisable to store patient information in an uncorrected form on the electronically 
secure servers of the information processor, using current digital security and encryption tools.

\section{Conclusion}

The study confirms the increasing importance of digital transformation in healthcare, while demonstrating the feasibility of using telemedicine technologies, implemented in a remote format, to provide medical care to patients with oesophageal hernia complicated by reflux esophagitis, living (staying, residing) in a locality remote from major cities of the Russian Federation. This normatively introduced approach to organising the digital paradigm of the doctor-patient relationship seems to be cost-effective and financially feasible, reducing and reducing the level of monetary costs. The use of a digital procedure for assessing patient data and medical parameters offers the possibility of extending the targeting of highly informative and high-tech radiological diagnostic techniques, while allowing correct and appropriate interpretation of results from specialists - radiologists who are constantly faced with assessing the symptomatology of such diseases. The main form of digital doctor-patient interaction is videoconferencing for consultation (case conference) to examine the patient's medical parameters recorded in electronic documentation, a conversation with the patient to clarify the nature of the individual pathological symptomatology. The use of this form of interaction makes it possible not only to determine the correct diagnosis in good time, but also to choose the best treatment tactics for the patient, including an assessment of the need for surgical intervention, the timeliness and promptness of which, in general, affects the patient's standard of living.

\section{References}

1. V.N Grigoryev, A.P. Sukhodolov, S.S. Ovanesyan, M.G. Spasennikova, V.V. Tyunkov, All-Russian Journal of Criminology 13(6), 873-883 (2019)

2. E. Gromova. T. Ivanc, BRICS Law Journal 7(2), 10$36(2020)$

3. O.V. Kamenskaya, I.Y. Loginova, A.S. Klinkova, A.R. Tarkova, R.A. Naydenov, E.I. Kretov, V.V. Lomivorotov, Russian Journal of Cardiology 25(6), 154-160 (2020)

4. T.S. Smal, V.D. Zavadovskaya, I.A. Deev, Bulletin of Siberian Medicine, 15(1), 79-88 (2016)

5. M.S. Zhuravlev, Law and information: issues of theory and practice: collected works of international scientific and practical conf, 151-154 (2017)

6. E.L. Nikonov, Surgery. Journal named after N.I. Pirogov 5, 96-105 (2018)

7. N.V. Gibadulin, I.O. Gibadulina, S.N. Trinov, Surgery. Journal named after N.I. Pirogov 11, 34-37 (2009)

8. O.N. Antropova, N.V. Pyrikova, I.V. Osipova, Russian Journal of Cardiology 7, 103-109 (2019)
9. G.A. Zhurbenko, A.S. Karpitsky, S.V. Panko, A.M. Shestyuk, Chest and Cardiovascular Surgery 57 (1), 36-42 (2015)

10. V.O. Shchepin, A.I. Bravve, A.I. Babenk, A.L. Tomchuk, Problems of Social Hygiene, Public Health and History of Medicine 5, 39-42 (2013)

11. M.D. Podolsky, A.A. Strokov, V.I. Kuznetsov, V.V. Shapovalov, Cardiovascular Therapy and Prevention 15 (5), 92-96 (2016)

12. V. A. Bondarenko, D.D. Kostoglodov, Modern problems of economics, law and business of postcoronavirus crisis. Proceedings of the International Scientific and Practical Online Conference, 114119 (2020)

13. I.B. Bashkova, T.V. Prokopyeva, A.V. Arkhipova, B.F. Ryapolov, Scientific and Practical Rheumatology 54 (1), 108 (2016)

14. M.V. Ionov, N.E. Zvartau, I.V. Emelianov, A.O. Konradi, Telemedical, Arterial Hypertension 25(4), 337-356 (2019)

15. D.N. Laptev, V.A Peterkova, Diabetes Mellitus 6, 420-426 (2017)

16. V.K. Fedyaeva, N.I. Zhuravlev, J.A. Galeeva, Diabetes Mellitus 4, 328-335 (2019)

17. Yu.N. Gorozhantsev, S.G. Sergienko, E.A. Vorotintseva, Yu.V. Emelianenko, Russian Journal of Cardiology 24, 39a-39b (2019) 